\title{
OriginaL
}

\section{ATF3 expression is induced by low glucose in pancreatic $\alpha$ and $\beta$ cells and regulates glucagon but not insulin gene transcription}

\author{
Yong-Soo Lee, Masaki Kobayashi, Osamu Kikuchi, Tsutomu Sasaki, Hiromi Yokota-Hashimoto,
} Vina Yanti Susanti, Yukari Ido Kitamura and Tadahiro Kitamura

Metabolic Signal Research Center, Institute for Molecular and Cellular Regulation, Gunma University, Maebashi, Gunma, Japan

\begin{abstract}
The pancreas is critical for maintaining glucose homeostasis. Activating transcription factor 3 (ATF3) is an adaptive response transcription factor. There are major discrepancies in previous reports on pancreatic ATF3; therefore, its role in the pancreas is unclear. To better elucidate the role of ATF3 in the pancreas, we conducted in vitro studies using pancreatic $\alpha$ and $\beta$ cell lines, and also evaluated the use of ATF3 antibodies for immunohistochemistry. We determined ATF3 expression was increased by low glucose and decreased by high glucose in both $\alpha$ TC-1.6 and $\beta$ TC 3 cells. We also showed that adenovirus-mediated ATF3 overexpression increased glucagon promoter activity and glucagon mRNA levels in $\alpha \mathrm{TC}-1.6$ cells; whereas, it had no effect on insulin promoter activity and insulin mRNA levels in $\beta \mathrm{TC} 3$ cells. Although immunostaining with the C-19 ATF3 antibody demonstrated predominant expression in $\alpha$ cells rather than $\beta$ cells, ATF3 staining was still detected in ATF3 knockout mice as clearly as in control mice. On the other hand, another ATF3 antibody (H-90) detected ATF3 in both $\alpha$ cells and $\beta$ cells, and was clearly diminished in ATF3 knockout mice. These results indicate that previous discrepancies in ATF3 expression patterns in the pancreas were caused by the varying specificities of the ATF3 antibodies used, and that ATF3 is actually expressed in both $\alpha$ cells and $\beta$ cells.
\end{abstract}

Key words: ATF3, Glucagon, Insulin, Pancreatic $\alpha$ cell, Pancreatic $\beta$ cell

ACTIVATING transcription factor 3 (ATF3) is a member of the ATF/CREB family of transcription factors. ATF3 gene expression is induced by a variety of extracellular signals, including cytokines, chemokines, growth factors, hormones, hypoxia, DNA damage, ER stress, and nutrient deprivation. Although ATF3 has long been recognized as a stress response gene, recent cumulative evidence suggests ATF3 is involved in more general adaptive responses, such as environmental, emotional, and nutritional alterations [1-3]. ATF3 is expressed in various cell types, and ATF3 disorders are associated with inflammatory diseases, immune diseases, and cancer [4].

To date, a number of in vitro and in vivo studies have been conducted to elucidate the physiological roles of ATF3 in the pancreas. However, there are major dis-

Submitted Sep. 17, 2013; Accepted Oct. 2, 2013 as EJ13-0383 Released online in J-STAGE as advance publication Oct.20, 2013

Correspondence to: Tadahiro Kitamura, M.D., Ph.D., Metabolic Signal Research Center, Institute for Molecular and Cellular Regulation, Gunma University, 3-39-15 Showa-machi, Maebashishi, Gunma 371-8512, Japan. E-mail: kitamura@gunma-u.ac.jp

(c) The Japan Endocrine Society crepancies in their findings. First, Wang et al. reported that ATF3 is present at higher levels in $\alpha$ cells than $\beta$ cells within islets [5]; however, Hartman et al. used double immunostaining to demonstrate clearly that ATF3-positive cells in islets correspond to insulin-positive but not glucagon-positive cells [6]. Second, in a cultured $\alpha$ cell line $(\alpha$ TC1.6), high glucose $(25 \mathrm{mM})$ reduced the level of ATF3 compared with low glucose $(2.5 \mathrm{mM})$ [7]. In contrast, the ATF3 level was higher under high-glucose conditions $(25-33 \mathrm{mM})$ than lowglucose conditions $(5-5.5 \mathrm{mM})$ in primary islets and a $\beta$ cell line (INS822/13) [6, 8]. Third, Wang et al. identified an ATF3 binding site in the glucagon promoter and showed that ATF3 activates glucagon transcription [7]; while, Zmuda et al. reported that ATF3 activates insulin1 transcription by binding to an ATF/CRE site on the insulin1 promoter [8]. The fourth discrepancy was whether the physiological role of ATF3 in the pancreas is beneficial or detrimental to the regulation of glucose metabolism. Both pancreas-specific (by Pdx1 promoter) and pancreatic $\beta$ cell-specific (by insulin promoter) ATF3 transgenic mice displayed reduced $\beta$ cell 
mass and severe glucose intolerance, indicating a deleterious function of ATF3 [6,9]. However, the same authors also reported that conventional ATF3 KO mice developed more severe glucose intolerance than control mice when fed a high fat diet as a result of decreased insulin gene transcription, suggesting that ATF3 has a beneficial function in the pancreas [8]. Together, these results suggest that ATF3 is a double-edged sword. We previously generated pancreas- and hypothalamus-specific ATF3 knockout mice and showed that these mice displayed better glucose tolerance and higher insulin sensitivity due to a lean phenotype; although, neither plasma insulin nor glucagon levels were changed [10].

In the present study, we demonstrate that ATF3 expression is increased by low glucose and decreased by high glucose in both $\alpha \mathrm{TC}-1.6$ and $\beta \mathrm{TC} 3$ cells; in addition, ATF3 regulates glucagon, but not insulin, transcription. We also demonstrated that the previous discrepancies in ATF3 expression patterns in the pancreas were caused by the different specificities of the ATF3 antibodies used. The C-19 antibody predominantly detected expression of ATF3 in $\alpha$ cells rather than $\beta$ cells; whereas, the $\mathrm{H}-90$ antibody detected ATF3 expression in both $\alpha$ cells and $\beta$ cells. Using pancreasspecific ATF3 KO mice, we showed that the C-19 antibody is less antigen-specific when used for immunohistochemistry, and that ATF3 is actually expressed in both $\alpha$ and $\beta$ cells in the mouse pancreas.

\section{Materials and Methods}

\section{DNA constructs, and adenoviruses}

We previously described the construction of pcDNA3.1-Flag-mATF3 and Ad-ATF3 [10]. pGL3mGlu (wt) and pGL3-mGlu (mt) harboring a mutated CRE/ATF binding site were gifts from D.F. Steiner (University of Chicago) [7]. pGL3-rIns containing 500 bp of the rat insulin1 promoter was provided by H.S. Choi (Chonnam National University).

\section{Cell culture, transfection, adenovirus infection, and luciferase assays}

The $\alpha \mathrm{TC}-1.6$ and $\beta \mathrm{TC} 3$ cells were maintained in high-glucose $(25 \mathrm{mM})$ or low-glucose $(2.5 \mathrm{mM})$ DMEM (Wako, Japan) supplemented with 2.5\% FBS (Gibco, USA) and $15 \%$ horse serum (Gibco, USA). All cell lines were cultured at $37^{\circ} \mathrm{C}$ in a humidified atmosphere containing $5 \% \mathrm{CO}_{2}$. Transient transfection was performed using Lipofectamine $2000^{\mathrm{TM}}$ (Invitrogen, USA) accord- ing to the manufacturer's instructions. Adenovirus infection was performed according to a standard protocol at a multiplicity of infection (MOI) of 50. For luciferase assays, the cells were co-transfected with a reporter plasmid and varying amounts of expression vectors. The total amount of DNA was adjusted to $0.8 \mu \mathrm{g} /$ well after the addition of each corresponding empty vector. The PRL-TK-Luc plasmid $(0.1 \mu \mathrm{g})$ was used as an internal control. The cells were harvested $24 \mathrm{~h}$ after transfection, and dual luciferase assays were performed. Each experiment was repeated at least three times.

\section{$R N A$ isolation and real time RT-PCR}

Total RNA was isolated from cells using RNAiso plus (Takara, Japan), and cDNAs was generated using the Improm II Reverse Transcription System (Promega, USA). The cDNA samples were then used for semi-quantitative RT-PCR or real time PCR using the Light Cycler system and Light Cycler 480 SYBR Green I (Roche, USA). The following primers were used: for ATF3, forward 5'-GGA GGC GGC GAG AAA GAA ATA-3' and reverse 5'-TCG GGG GCA GAG TGG GTC AAT-3'; for Glucagon, forward 5'-ATG AAG ACC ATT TAC TTT GTG GCT G-3' and reverse 5'-CGG CCT TTC ACC AGC CAC GC-3'; for Insulin1, forward 5'-TAG TGA CCA GCT ATA ATC AGA G-3' and reverse 5'-ACG CCA AGG TCT GAA GGT CC-3'; and for GAPDH, forward 5'-TCC ACC ACC CTG TTG CTG TAG-3' and reverse 5'-GAC CAC AGT CCA TGC CAT CAC-3'.

\section{Western blot analyses}

Cell lysates were prepared using lysis buffer $(50 \mathrm{mM}$ Tris $\mathrm{pH} 8.0,150 \mathrm{mM} \mathrm{NaCl}, 10 \%$ glycerol, $1 \%$ Triton $\mathrm{X}-100,0.1 \%$ SDS, $5 \mathrm{mM}$ EDTA, $1 \mathrm{mM}$ DTT, $10 \mathrm{mM}$ $\mathrm{NaF}, 1 \mathrm{mM} \mathrm{Na} \mathrm{VO}_{4}$ ) supplemented with a Completed Mini protease inhibitor cocktail tablet (Roche Diagnostics, Tokyo, Japan). Proteins from whole cell lysates were separated by $15 \%$ SDS-PAGE and transferred to BioTrace ${ }^{\mathrm{TM}}$ NT nitrocellulose transfer membranes (Pall Life Sciences, USA). The membranes were probed with mouse anti-ATF3 monoclonal antibody. Mouse monoclonal antibodies against human/ mouse ATF3 were described previously [10]. To determine the relative amounts of ATF3 in each experiment, membranes were re-probed with anti-B23 (Santa Cruz, USA). Immunoreactive proteins were visualized using an Immobilon Western chemiluminescent HRP substrate (Millipore, USA) and the LAS-3000 Image ana- 
lyzer (Fuji Film, Japan).

\section{Animals}

The generation of pancreas- and hypothalamus-specific ATF3 KO mice was described previously [10]. Mice were housed in individual cages in a temperaturecontrolled facility with a 12-h light, 12 -h dark cycle. All animal care and experimental procedures were approved by the Institutional Animal Care and Experimentation Committee at Gunma University. All animal experimentation described in the manuscript was conducted in accordance with accepted standards of humane animal care, as outlined in the ethical guidelines.

\section{Immunohistochemistry}

The immunohistochemistry of fixed-frozen sections $(0.5 \mu \mathrm{m}$ thick) of mouse pancreas was performed with anti-ATF3 (C-19 or H-90) (Santa Cruz, USA), antiglucagon (Sigma, USA), and anti-insulin antibodies (Dako, USA). We visualized immune complexes with FITC- or Cy3-conjugated secondary antibodies. We analyzed at least 10 sections for each mouse, and six mice for each genotype.

\section{Statistical analysis}

We performed descriptive statistics and ANOVA followed by Fisher's test using the Statview software package (Abacus Concepts).

\section{Results}

\section{ATF3 expression is increased by low glucose and decreased by high glucose in both $\alpha$ TC-1.6 and $\beta T C 3$ cells}

Wang et al. previously reported that ATF3 expression is induced by low glucose and regulates glucagon transcription in pancreatic $\alpha$ cells [7]. Consistent with this report, we found, using semiquantitative RT-PCR analysis, that ATF3 expression was increased by low glucose $(2.5 \mathrm{mM})$ and decreased by high glucose $(25 \mathrm{mM})$ conditions in $\alpha \mathrm{TC}-1.6$ cells (Fig. 1A). We confirmed these results at the protein level by demonstrating ATF3 protein levels were increased by low glucose (Fig. 1C). In contrast to $\alpha$ cells, ATF3 expression in $\beta$ cells is reportedly increased by high glucose and decreased by low glucose conditions $[6,8]$. However, we clearly showed that ATF3 expression was also increased by low glucose and decreased by high glucose in $\beta$ TC3 cells (Fig. 1B). When we performed real-time RT-PCR to quantify these results, we found that low-glucose culture conditions increased ATF3 mRNA 25-fold and high glucose conditions reduced it by $60 \%$ (Fig. 1D). It is notable that these changes in ATF3 expression were reversible: $24 \mathrm{~h}$ high glucose after $24 \mathrm{~h}$ in low-glucose culture and $24 \mathrm{~h}$ low glucose after $24 \mathrm{~h}$ in high-glucose culture restored the expression levels of ATF3 (Fig. 1D). These results indicate that ATF3 expression is increased by low glucose and decreased by high glucose in both $\alpha$ and $\beta$ cells.

\section{ATF3 regulates glucagon gene transcription in $\alpha T C$ - 1.6 cells, but does not regulate insulin gene transcrip- tion in $\beta$ TC3 cells}

Luciferase assays revealed that glucagon promoter activity was increased by ATF3 expression in a dosedependent manner, while ATF3 expression had no effect on the mutant glucagon promoter in which the ATF/CRE site was altered (Fig. 2A). We also observed increased glucagon mRNA levels in aTC-1.6 cells infected with an ATF3-expressing adenovirus (Fig. 1C). However, ATF3 overexpression had no discernible effect on either insulin 1 promoter activity or insulin 1 mRNA levels in $\beta$ TC3 cells (Fig. 2B, D), despite the presence of an ATF/CRE site in the insulin1 promoter used. These results are inconsistent with those of previous studies $[6,8]$.

\section{ATF3 is expressed in both $\alpha$ and $\beta$ cells in the mouse pancreas}

The most critical discrepancy in previous reports was whether ATF3 was expressed in $\alpha$ or $\beta$ cells in islets. Although Wang et al. reported that ATF3 is present at a higher level in $\alpha$ cells than $\beta$ cells [5], Hartman demonstrated that ATF3-positive cells correspond to insulin-positive but not glucagon-positive cells [6]. We attempted to clarify this point by comparing the pancreatic expression patterns of ATF3 in wild-type and pancreas-specific ATF3 KO mice, using two kinds of antibodies against ATF3 (C-19 and H-90). As shown in Fig. 3A, double immunostaining with the C-19 antibody and anti-glucagon or anti-insulin antibody indicated that ATF3 is predominantly expressed in $\alpha$ cells rather than $\beta$ cells. However, ATF3 staining was also detected in ATF3 KO mice as clearly as in the control mice (Fig. 3B). In contrast, as we reported previously, the staining with the H-90 antibody revealed ATF3 was expressed in both $\alpha$ and $\beta$ cells, and was undetectable in ATF3 KO mice [10]. These results indicate that the H-90 antibody provides more reliable staining results than the C-19 antibody, and that ATF3 is actually 
A
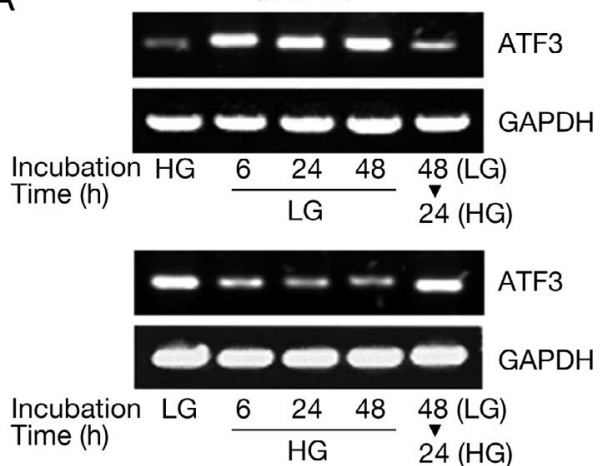

C

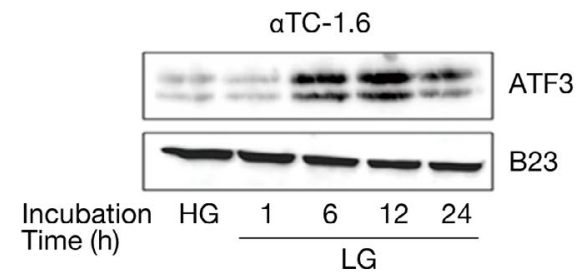

B

BTC3
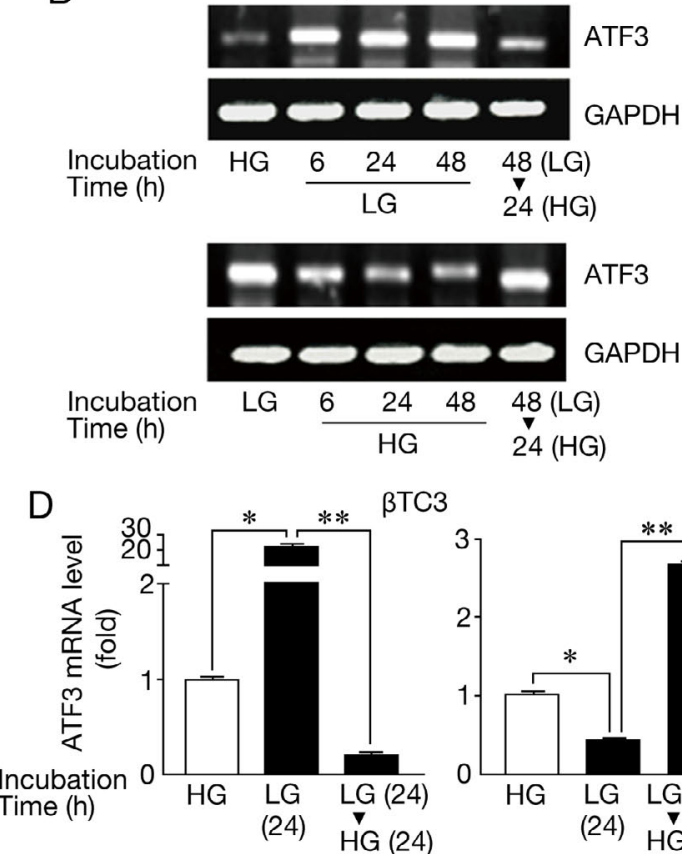

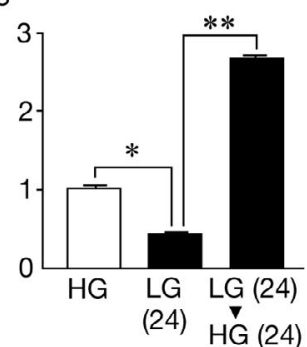

Fig. 1 ATF3 expression is increased by low glucose and decreased by high glucose in both $\alpha \mathrm{TC}-1.6$ cells and $\beta$ TC 3 cells. (A and C) $\alpha$ TC-1.6 cells and (B and D) $\beta T C 3$ cells were cultured with high $(25 \mathrm{mM})$ or low $(2.5 \mathrm{mM})$ glucose for more than 4 weeks, and then cultured with either low or high glucose for the indicated periods. In some cases, after $48 \mathrm{~h}$ culture with low or high glucose, the medium was replaced with high or low glucose, respectively, and the cells were cultured for an additional $24 \mathrm{~h}$. (A and B) Total RNAs was isolated from the harvested cells and used for RT-PCR to assess ATF3 levels. (C) The ATF3 protein levels were analyzed by Western blotting with anti-ATF3 monoclonal antibody. (D) ATF3 mRNA levels were analyzed by realtime RT-PCR. The results were normalized using GAPDH. Data represent the mean \pm SEM fold increase relative to controls. $* P<0.05, * * P<0.01$ by ANOVA.

A
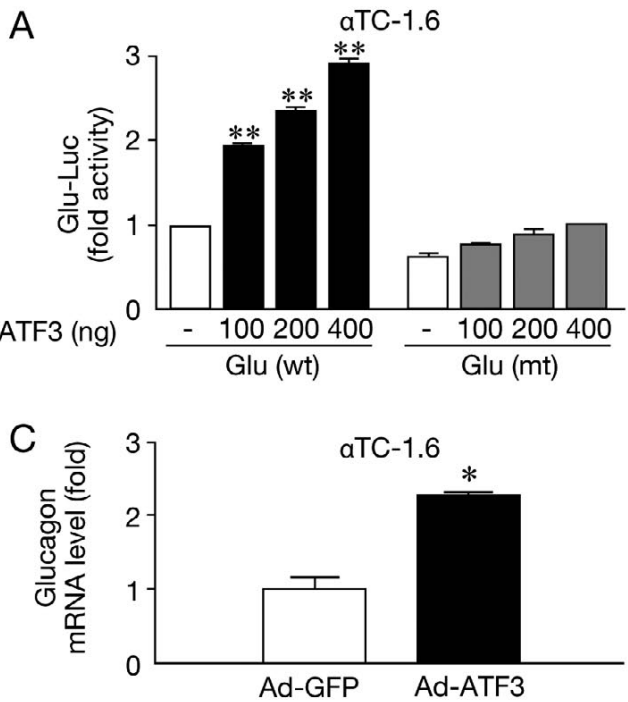

B
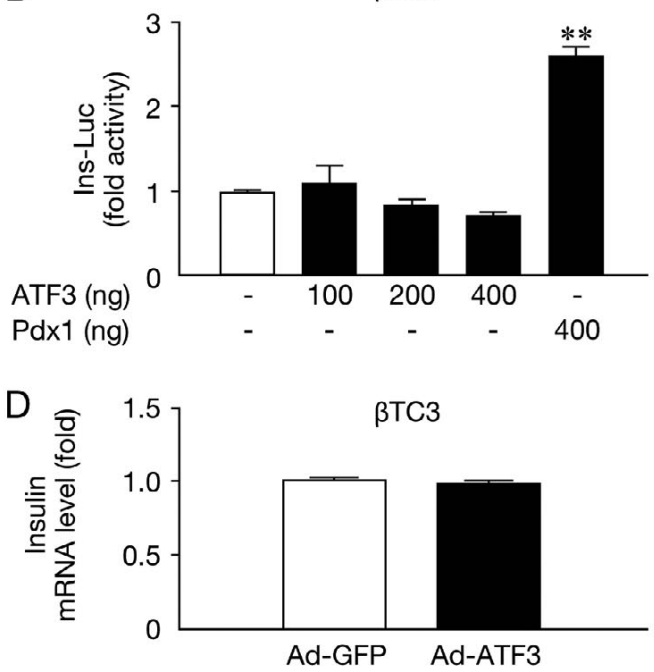

Fig. 2 ATF3 regulates glucagon but not insulin gene transcription.

(A) Glucagon and (B) insulin1 promoter-driven luciferase activities were measured in $\alpha \mathrm{TC}-1.6$ and $\beta \mathrm{TC} 3$ cells, respectively, transfected with the indicated amount of pcDNA-Flag-mATF3 DNA. For (A), we used a mutant glucagon promoter that harbors a mutated ATF/CRE site as a negative control. For (B), we used Pdx1 as a positive control. (C) $\alpha \mathrm{TC}-1.6$ cells and (D) $\beta T C 3$ cells were infected with adenoviruses expressing ATF3 (Ad-ATF3); $24 \mathrm{~h}$ later, mRNA was isolated from the cells and used for real-time RT-PCR to quantify glucagon and insulin mRNA levels, respectively. Data represent the mean \pm SEM fold increase relative to controls. $* P<0.05, * * P<0.01$ by ANOVA. 
A
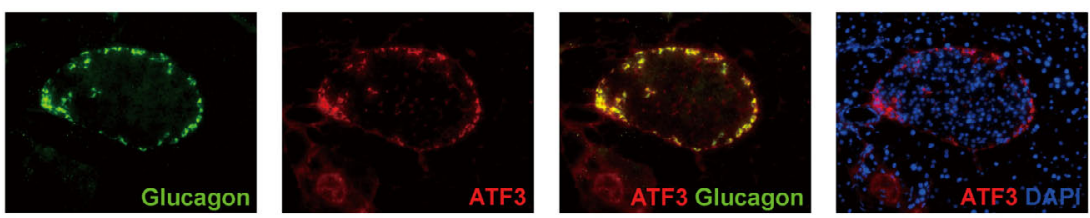

(C-19)
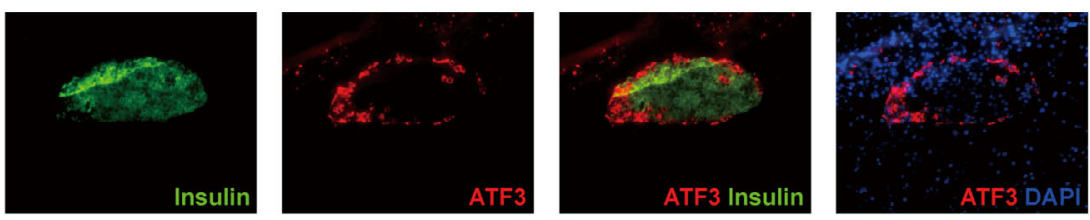

B
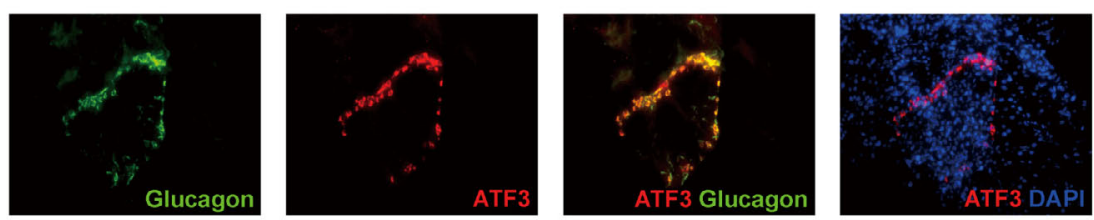

$(\mathrm{C}-19)$
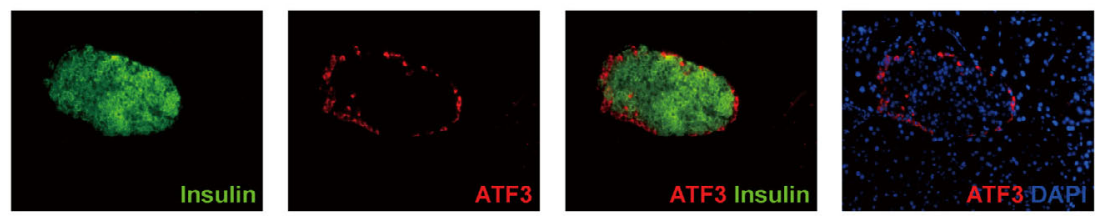

Fig. 3 ATF3 immunoreactivity with the C-19 antibody in mouse pancreatic $\alpha$ cells is not diminished in ATF3 KO mice.

Frozen-sectioned pancreata from pancreas- and hypothalamus-specific ATF3 KO and control mice were immunostained with anti-ATF3 (C-19) antibody followed by Cy3-conjugated secondary antibody (red), along with anti-glucagon or anti-insulin antibody followed by fluorescein isothiocyanate-conjugated secondary antibody (green) and DAPI staining (blue); 200× magnification. Merged images are shown in some panels. Note, this finding was in contrast to the previous results with H-90 antibody, in which ATF3 immunoreactivity was clearly diminished in ATF3 knockout mice [10].

expressed in both $\alpha$ and $\beta$ cells in the mouse pancreas. However, C-19 antibody is raised against C-terminal 19 amino acids of human ATF3, while H-90 antibody is raised against $\mathrm{N}$-terminal 90 amino acids of human ATF3, therefore another possibility for the different performances of these antibodies in ATF3 $\mathrm{KO}$ mice is that C-19 antibody may detect the alternative splicing variant of ATF3.

\section{Discussion}

There are several contradictions in published reports regarding the physiological role of ATF3 in the pancreas, and some serious issues remain unsolved. We attempted to elucidate these critical issues in this study. Wang et al. showed that ATF3 is expressed more in $\alpha$ cells than in $\beta$ cells [5], yet, Hartman et al. reported that ATF3 is exclusively expressed in $\beta$ cells, not in $\alpha$ cells [6]. We previously demonstrated that ATF3 is actually expressed in both $\alpha$ and $\beta$ cells by immunostaining with the H-90 ATF3 antibody [10]. We determined here that this discrepancy was caused by the different specifici- ties of the ATF3 antibodies used in those studies. The presence of ATF3 staining in the ATF3 knockout islets and the unexpected cytoplasmic ATF3 staining (Fig. 3) suggested the C-19 antibody is less antigen-specific than the H-90 antibody when used in immunohistochemistry. Hai et al. also recently pointed out this problem with the C-19 antibody [11]. Although there are several antibodies against ATF3 available commercially, their varying performances may lead to contrasting pancreatic expression patterns of ATF3.

The second discrepancy was whether ATF3 expression is induced by low glucose [7] or high glucose $[6,8]$ in $\alpha$ and $\beta$ cells. Our results in $\alpha \mathrm{TC}-1.6$ and $\beta \mathrm{TC} 3$ cells clearly demonstrated that ATF3 expression is induced by low glucose in both $\alpha$ and $\beta$ cells (Fig. 1). These results are consistent with Wang's report, but are inconsistent with two other papers $[6,8]$. Although we still do not know the reason for this apparent discrepancy, it may be due to the different $\beta$ cell lines used in these studies.

The third critical issue was the role of ATF3 with respect to glucagon or insulin promoters. Although the proximal regions of the glucagon and insulin1 promot- 
ers each contain a canonical ATF/CRE site, luciferase assays in this study revealed that ATF3 regulates glucagon promoter activity but not insulin1 promoter activity (Fig. 2A, B). Quantification of glucagon and insulin 1 mRNA in ATF3-overexpressing $\alpha$ TC-1.6 and $\beta$ TC3 cells also supported this conclusion (Fig. 2C, D).

The final issue was whether pancreatic ATF3 is beneficial or detrimental to the regulation of glucose homeostasis. It is actually highly controversial whether ATF3 in $\beta$ cells is beneficial (by enhancing insulin gene transcription) or deleterious (by inducing apoptosis via suppression of IRS2 gene transcription). Nonetheless, our previous study showed that pancreas- and hypothalamus-specific ATF3 KO mice have normal serum glucagon and insulin levels, unchanged glucose-responsive glucagon and insulin secretion, and normal $\alpha$ cell- and $\beta$ cell-masses [10]. These results suggested that the physiological role of ATF3 in the pancreas is less significant than previously thought $[6,8,9]$. However, we still do not know the reason for the discrepancy between in vitro and in vivo results regarding the effect of ATF3 on glucagon. Hypoglycemia presents an immediate threat to survival; therefore, other factors may compensate for plasma glucagon levels in vivo.

In conclusion, the discrepancies regarding ATF3 in previous studies might have been caused by the different specificities of the ATF3 antibodies (especially when used for immunohistochemistry). In our previous study, we used pancreas- and hypothalamus-specific ATF3 KO mice; therefore, the phenotypes of the mice were mainly due to the ATF3 deficiency in the hypothalamus. Thus, future studies are necessary to elucidate the physiological role of ATF3 in $\alpha$ and $\beta$ cells in vivo using $\alpha$ cell- or $\beta$ cell-specific ATF3 knockout mice.

\section{Acknowledgments}

This study was supported by Grants-in-Aid for Scientific Research (B) to T.K. In addition, this study was supported in part by the Joint Usage/Research Program of the Medical Research Institute, Tokyo Medical and Dental University. We thank C. Osawa for excellent technical assistance and the members of the Kitamura lab for helpful discussions.

\section{Disclosure}

There are no conflicts of interest relevant to this article.

\section{References}

1. Montminy M (1997) Transcriptional regulation by cyclic AMP. Annu Rev Biochem 66: 807-822.

2. Hai T, Hartman MG (2001) The molecular biology and nomenclature of the activating transcription factor/ cAMP responsive element binding family of transcription factors: activating transcription factor proteins and homeostasis. Gene 273: 1-11.

3. Green TA, Alibhai IN, Unterberg S, Neve RL, Ghose $\mathrm{S}$, et al. (2008) Induction of activating transcription factors (ATFs) ATF2, ATF3, and ATF4 in the nucleus accumbens and their regulation of emotional behavior. J Neurosci 28: 2025-2032.

4. Hai T, Wolford CC, Chang YS (2010) ATF3, a hub of the cellular adaptive-response network, in the pathogenesis of diseases: is modulation of inflammation a unifying component? Gene Expr 15: 1-11.

5. Wang J, Webb G, Cao Y, Steiner DF (2003) Contrasting patterns of expression of transcription factors in pancreatic alpha and beta cells. Proc Natl Acad Sci U S A 100: 12660-12665.

6. Hartman MG, Lu D, Kim ML, Kociba GJ, Shukri T, et al. (2004) Role for activating transcription factor 3 in stress-induced beta-cell apoptosis. Mol Cell Biol 24:
5721-5732.

7. Wang J, Cao Y, Steiner DF (2003) Regulation of proglucagon transcription by activated transcription factor (ATF) 3 and a novel isoform, ATF3b, through the cAMP-response element/ATF site of the proglucagon gene promoter. J Biol Chem 278: 32899-32904.

8. Zmuda EJ, Qi L, Zhu MX, Mirmira RG, Montminy MR, et al. (2010) The roles of ATF3, an adaptive-response gene, in high-fat-diet-induced diabetes and pancreatic beta-cell dysfunction. Mol Endocrinol 24: 14231433.

9. Li D, Yin X, Zmuda EJ, Wolford CC, Dong X, et al. (2008) The repression of IRS2 gene by ATF3, a stressinducible gene, contributes to pancreatic beta-cell apoptosis. Diabetes 57: 635-644.

10. Lee YS, Sasaki T, Kobayashi M, Kikuchi O, Kim HJ, et al. (2013) Hypothalamic ATF3 is involved in regulating glucose and energy metabolism in mice. Diabetologia 56: 1383-1393.

11. Hai T, Jalgaonkar S, Wolford CC, Yin X (2011) Immunohistochemical detection of activating transcription factor 3, a hub of the cellular adaptive-response network. Methods Enzymol 490: 175-194. 